\title{
Designing a Story Database for Use in Automatic Story Generation
}

\author{
Katri Oinonen ${ }^{1}$, Mariët Theune ${ }^{1}$, Anton Nijholt ${ }^{1}$, and Jasper Uijlings ${ }^{2}$ \\ ${ }^{1}$ University of Twente PO Box 217 \\ $7500 \mathrm{AE}$ Enschede \\ The Netherlands \\ $\{$ k.m.oinonen I m.theune I a.nijholt $\} @$ ewi.utwente.nl \\ 2University of Amsterdam \\ PO Box 19268 \\ 1000 GG Amsterdam \\ The Netherlands \\ jrruijli@science.uva.nl
}

\begin{abstract}
In this paper we propose a model for the representation of stories in a story database. The use of such a database will enable computational story generation systems to learn from previous stories and associated user feedback, in order to create believable stories with dramatic plots that invoke an emotional response from users. Some of the distinguishing characteristics of our proposal are the inclusion of what we call 'narratological concepts' and user feedback in the story database.
\end{abstract}

Keywords: Story database, story representation, automatic story generation.

\section{Introduction}

In this paper we present our ideas about designing a story database which should enable a computational story generation system to learn from annotated representations of existing stories (either human-authored or automatically generated). The story structures represented in the story database can be used by reasoning algorithms for story generation, transforming them for use in new stories. We propose to use a much more expressive knowledge representation model for stories than the representation used in the MINSTREL system of [1] and the KIIDS system of [2]. In MINSTREL a database of story schemas including character goals, actions, and the states of objects, together with knowledge about dramatic writer goals, is used for case-based creative problem solving to generate stories. KIIDS is based on a similar case-based reasoning process as MINSTREL, and uses a case-base of stories built upon a story knowledge ontology related to three basic domains: interactive goaldirected experiences, narrations, and simple simulation. We argue that the story representation model should not only include detailed information about story content linked to the temporal representation of the whole story, but also meta knowledge about which ingredients make up a good story, which we call narratological concepts. 
We also propose to annotate the stories in the database with user feedback about their emotional impact, so that an interactive automated story generation system can learn from these annotations to construct captivating stories during interaction with a user.

\section{General Requirements for Story Representation}

In our model for story representation we distinguish five related levels, representing different story aspects. In the first two levels the basic story elements, such as the characters, objects, and environment involved in the story are described with their properties and relationships. The semantics of the stories is captured by linking the story elements to the actions, events, and background processes in the evolving story plot represented in the third level. In addition, in the last two levels our model should include meta-knowledge about story structure, which we call narratological concepts, and user feedback about emotional impact.

Level 1: Semantic networks for story world knowledge. As a basis for story representation we need a basic general ontology: a semantic network describing the knowledge about the concepts and the possible relations between them in the story domain (see e.g., [3]). A story world description, or world state, is a specific instantiation of (some of) the concepts from the general ontology at a certain point in time, together with the current values of their properties and relationships.

Level 2: Character representations. A complex and extensive representation of intelligent characters acting a role in the story is needed. The emotional reaction of the user to what happens to the characters in the story will depend greatly on the emotional-relational properties of these characters. At least information about the characters' physical state, perceptions, beliefs, personality, emotions, social emotional relationships, desires and motivations, action potential, coping strategy, goals, and action plans should be represented in the character model. Gratch and Marsella [4] use similar concepts to simulate the generality of human emotional capabilities, such as appraisal and coping strategies: how characters assess what they perceive, and how they react to this assessment.

Level 3: Plot structures as a causal and temporal network. We propose to represent the stories and story fragments in the database as causal network or graph models of actions and events, linked to the representations of the characters and objects that play a role in them [5]. Actions are intentionally performed by the characters in the story, whereas we define events as changes in the world that are not the direct and planned result of any character action. The causal network should also be annotated with time, allowing us to track how the properties of characters and objects change in subsequent world states as a consequence of the actions and events occurring in the story world, as well as background processes that simulate the life, physics, biology, and chemistry in the story world environment. 
Level 4: Representation of narratological concepts. To enable a computational system to reason about which ingredients make up a dramatic plot, the story representation model also needs to include narratological concepts: meta-knowledge about story structures and their narrative properties. Relevant narratological concepts include coherence, relevance, and conflict. These concepts can be represented as nary relationships linking elements from the network representation of the plot (Level 2). Knowledge of these relationships can be used by pattern matching algorithms for detecting dramatic emotional story structures, and for creating new stories by manipulating the structures found in previous stories. We propose to measure the degree of coherence of a story by the number of related objects and events covered in a consistent representation of the whole plot. This is similar to the quantitative measure thickness of narrative plot structures proposed by [6], and corresponds to the complexity / richness of the whole story. In our model, the relevance of an action, event or background process in a story can be measured from the perspective of a character, a community in the story world, or a user. The degree of relevance depends on (1) the strength and importance of its effect on the story world, (2) the number, strength, and importance of the causally or semantically related elements involved with it, (3) the current goals and motivations of the involved characters. This information can be combined with user feedback annotations (Level 5) to determine relevance. In a conflict, at least two characters share a contradicting desire about the state of the same object or about their relationship with the object.

Level 5: Representation of the cognitive emotional interactor. The story representation model should also include information about the story or story fragments' potential effects (e.g., suspense) on the user, based on empirically collected user feedback. Then, by automated detection of patterns of narratological concepts that caused certain effects on the user [7] in past stories, the system will be able to predict the audience response to new stories. Later, the potential effects can be derived from how, during interactive story generation, users influence the course of the story from an outsider or a character perspective.

\section{Using a Story Database in the Virtual Storyteller}

The Virtual Storyteller ${ }^{1}$ is a multi-agent framework where a story plot is generated using autonomous Character Agents capable of goal-oriented action planning, and by a Plot Agent that steers the emerging plot by (among other things) influencing the Characters' perceptions and manipulating the story world. The resulting plot is represented as a structure of causally linked events, and converted to natural language. The Plot Agent has not been fully implemented yet; an implementation of the Character Agents is described in [8].

Although the Virtual Storyteller does not include a story database yet, much of the information required to develop such a database is already available in the system. Corresponding to Level 1 the Virtual Storyteller makes use of ontologies for plot

\footnotetext{
${ }^{1} \mathrm{http}: / / \mathrm{ww} w h o m e . c s . u t w e n t e . n l / \sim$ theune/VS/index.html
} 
creation: 1) a basic general ontology defining a classification of objects, and 2) an Action Ontology defining a set of actions that the Character Agents can undertake in the story world, both represented in the W3C standard Web Ontology Language (OWL). See [9] for more details. In addition, the World Agent maintains a story world description (world state): an instantiation of the objects which currently are present in the environment, their properties and the relationships between them. In [8] we describe an initial implementation of the Character Agents used in the Virtual Storyteller that includes the main concepts from Level 2 of our story representation model: beliefs, goals, actions plans, personality and emotions. Our emotion model incorporates appraisal and coping, specifying how, depending on personality, the Character Agents' emotions are influenced by what happens in the story world, and how their actions are influenced by these emotions. Corresponding to Level 3, the Virtual Storyteller uses a causal plot representation which is an adapted version of the representation proposed by [5]. It includes actions, events, perceptions, goals, goal outcomes, and character 'internal elements' such as emotions and beliefs, linked by motivation, enablement, mental and physical cause relations and annotated with temporal information. See [10] for more details. Levels 4 and 5, the narratological concepts and user feedback annotation, are not yet included in our system but are the subject of future work.

Acknowledgments. The authors are involved in the EU Network of Excellence HUMAINE (Human-Machine Interaction Network on Emotion).

\section{References}

1. Turner, S.: The Creative Process: A Computer Model of Storytelling. Lawrence Erlbaum Associates, Hillsdale, NJ (1994)

2. Peinado, F., Gervás, P.: Creativity Issues in Plot Generation. Working Notes of the IJCAI05 Workshop on Computational Creativity (2005) 45-52. Technical Report 5-05.

3. Reithinger, N., Pecourt, E., Nikolova, M.: Meta-Data for Interactive Storytelling. Proceedings International Conference on Virtual Storytelling (2005) 172-175

4. Gratch, J., Marsella, S.: A Domain-Independent Framework for Modeling Emotion. Journal of Cognitive Systems Research 5-4 (2004) 269-306

5. Trabasso, T., van den Broek, P., Suh, S.: Logical Necessity and Transitivity of Causal Relations in Stories. Discourse Processes 12 (1989) 1-25

6. Alterman, R., Bookman, L.A.: Some Computational Experiments in Summarization. Discourse Processes 13 (1994) 143-174

7. Szilas, N.: IDTension: A Narrative Engine for Interactive Drama. Proceedings of the Technologies for Interactive Digital Storytelling and Entertainment (TIDSE) Conference (2003) 187-203

8. Theune, M., Rensen, S., op den Akker, R., Heylen, D., Nijholt, A.: Emotional characters for automatic plot creation. Proceedings of the Technologies for Interactive Digital Storytelling and Entertainment (TIDSE) Conference, Lecture Notes in Computer Science Vol. 3105 (2004) 95-100

9. Uijlings, J.: Designing a Virtual Environment for Story Generation. MSc thesis, University of Amsterdam, Amsterdam, The Netherlands (2006)

10. Swartjes, I.: The Plot Thickens: Bringing Structure and Meaning into Automated Story Generation. MSc thesis, University of Twente, Enschede, The Netherlands (2006) 